creatine kinase concentration in conjunction with substantial concentrations of MB isoenzymes, elevated aspartate aminotransferase and lactate dehydrogenase in the setting of normal liver function. One-third of the patients had plasma free carnitine concentration below $20 \mathrm{mamol} / \mathrm{L}$, a concentration associated with depletion of carnitine in tissues. Linear growth decreased after one year of age and head circumferences, normal in the first three to four years of life, then fell to the third percentile in mid-childhood. Longevity was reduced because of renal disease and the oldest patient examined was 33 years. (Charnas LR, Gahl WA et al. Clinical and laboratory findings in the oculocerebrorenal syndrome of Lowe, with special reference to growth and renal function. N Engl J Med May 9, 1991; 324:1318-25).

COMMENT. The oculocerebrorenal syndrome of Lowe, an $\mathrm{X}$-linked recessive disorder, is a rare cause of neonatal hypotonia or the limp infant syndrome. Both muscle and central abnormalities may contribute to the hypotonia and areflexia.

\title{
PREDNISONE-TREATED DUCHENNE MUSCULAR DYSTROPHY
}

The immosuppressant/antiinflammatory effects of prednisone were studied in 33 patients with Duchenne muscular dystrophy (ages 5 to 15 years) at the conclusion of a six month treatment trial at the Department of Neurology, Division of Neuromuscular Disease, Ohio State University College of Medicine, Columbus, OH. Immunohistochemical analyses were carried out on muscle biopsies: 12 from the placebo group, 9 from the low-dose prednisone group $(0.75 \mathrm{mg} / \mathrm{kg} / \mathrm{d})$, and 12 from the high-dose group $(1.5 \mathrm{mg} / \mathrm{kg} / \mathrm{d})$. The number of $\mathrm{T}$ cells and the number of muscle fibers focally invaded by $\mathrm{T}$ cells were significantly decreased in the prednisone treated groups compared with controls. Prednisone may improve strength in Duchenne muscular dystrophy through primarily immunologic mechanisms involving $\mathrm{T}$ lymphocytes. (Kissel JT et all. Mononuclear cell analysis of muscle biopsies in prednisone-treated and untreated Duchenne muscular dystrophy. Neurolo-

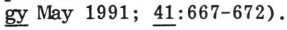

COMMENT. The possibility that clinical improvement was related to prednisone-induced alterations in skeletal muscle dystrophin was disproved by a further study from Ohio State University (Burrow $\mathrm{KL}$ et al. Dystrophin expression and somatic reversion in prednisone-treated and untreated Duchenne dystrophy. Neurology May $1991 ; 41: 661-666)$. Dystrophin content was analyzed at the conclusion of a six month trial of prednisone, using Western blots and antibody staining of tissue sections. There was no significant differences in dystrophin content between treatment and placebo groups.

\section{INFECTIOUS DISEASES}

\section{EPIDEMIOLOGY OF ENCEPHALITIS}

The incidence and epidemiology of encephalitis in children have been analyzed in a 20 year survey at the Department of Virology and the 
Children's Hospital, University of Helsinki, Finland. There were 405 patients with an overall male to female ratio of 1.4:1. Patients were grouped according to age: One to 11 months (33), one to four years (139), five to nine years (159), and ten to 16 years (74). The etiology was determined in $69 \%$; it was unknown among one-half of the youngest children and in only one-fourth of those at least ten years of age. The incidence was highest in children younger than two years (16.7/100,000 child years) and was very low in those 14 to 16 years of age $(1 / 100,000$ child years). After the introduction of vaccination against mumps, measles and rubella in 1983, the major encephalitides have been varicella-zoster, Mycoplasma pneumoniae, and respiratory and enteroviruses. Enteroviruses and Herpes simplex were most common in the infants younger than one year of age whereas respiratory viruses and Mycoplasma pneumoniae as well as varicella-zoster dominated in older children. Mumps and varicella were more cormon in boys, and adenovirus and Mycoplasma pneumoniae were more cormon in girls. Seasonal accumulation appeared in February and March and the incidence was lower in July and August. (Koskiniemi M et al. Epidemiology of encephalitis in children: a 20-year survey. Ann Neurol May 1991; $\underline{29}: 492-497$ ).

COMMENT. This study is important in emphasizing a greater interest in childhood encephalitis and the need for early diagnosis and treatment especially in young infants. The Mayo Clinic study in 1984 had shown that encephalitis in young children is often diagnosed late. The authors suggest that convulsions of unknown origin may be due to ill-defined encephalitis caused by respiratory viruses and adenovirus in winter months and enteroviruses in autumn. The etiology is often difficult to establish but antigen tests for respiratory viruses, adenoviruses, Herpes virus, and Mycobacterium pneumoniae are available and will provide the diagnosis within a few hours.

\section{POSTENCEPHALITIC TOURETTE SYNDROME}

A six year old girl who developed a Tourette-like syndrame following herpes encephalitis is reported from the Division of Child Neurology, Walter Reed Army Medical Center, Washington, DC and Departments of Neurology and Pediatrics, Johns Hopkins University School of Medicine, Baltimore, MD. A three day prodrame of headaches, malaise, nausea and vomiting was followed by fever and focal motor seizures involving the left side of the face and the left arm. Lumbar puncture showed 122 IBCs ( $80 \%$ lymphocytes) and a protein of $31 \mathrm{mg} / \mathrm{dl}$. A repeat lumbar puncture within 24 hours showed $750 \mathrm{WBCs}, 43 \mathrm{RBCs}$, and protein $85 \mathrm{mg} / \mathrm{dl}$. CT on hospital day three and subsequent MRIs showed a small hemorrhagic lesion in the right mesial temporal region and progressive edematous changes in the right temporal lobe, basal ganglia and thalamus. EEG showed focal slowing over the right temporal and frontal lobes. A presumptive diagnosis of herpes encephalitis was made and Acyclovir was given for ten days. At two weeks after discharge she developed eye blinking and sudden rapid recurrent purposeless nonrhythmic stereotyped motor movements and vocalizations. The tics 